Indian Journal of Medical Sciences

(INCORPORATING THE MEDICAL BULLETIN)

\begin{tabular}{ccc|}
\hline VOLUME 62 & AUGUST 2008 & NUMBER 8 \\
\hline & EDITORIALS & \\
\hline
\end{tabular}

\title{
WHAT IS THE USE OF ROUTINE ENDOTRACHEAL SURVEILLANCE CULTURES IN VENTILATED PATIENTS?
}

As the most common nosocomial infection on intensive care units, there is no doubt that ventilator associated pneumonia (VAP) has a remarkable clinical and financial impact on health care systems these days. Thus, it is no wonder that various efforts are being made to improve the outcome of this type of infection. In many facilities this includes the routine diagnostic sampling of endotracheal aspirate (ETA) of patients who have not yet developed pneumonia. There are two aims of this practice: the first one is to assess the current colonization status of each individual patient. In the case that VAP is suspected at a later time the most appropriate antimicrobial therapy could then be applied immediately and chances for an improved clinical course of disease increase. ${ }^{[1]}$ The second aim of routine ETA sampling is to avoid the use of empiric broad spectrum antibiotic treatment which in consequence may lead to selection of highly resistant pathogens over time. But is this procedure really helpful in the identification of microorganisms that cause the infection later during the stay of the patient? This issue is causing controversial discussion these days since the overall number of studies that address this topic is still low.
In 1997 Delclaux et al, ${ }^{[2]}$ obtained repeated protected specimens from the lower respiratory tracts of 30 patients suffering from severe adult respiratory distress syndrome (2 to 20 serial samplings per patient). 24 episodes of VAP were diagnosed in 18 patients (4.2 per 1,000 ventilation days). The overall positive predictive value (PPV) of prior lower respiratory tract colonization for subsequent infection was 0.89 , but was microbiologically confirmed in 16 of the $24(67 \%)$ cases of VAP only. Nevertheless, the negative predictive value (NPV) in this study was no more than 0.60 .

More recently Michel and coworkers ${ }^{[3}$ performed a prospective observational study on 299 mechanically ventilated patients in a medical intensive care unit (ICU). Routine ETA sampling was done twice a week in all of them. When VAP was suspected in a patient, bronchoalveolar lavage (BAL) was carried out for diagnostic purpose. BAL became necessary for 75 of the 299 patients during this study. VAP was confirmed by BAL cultures in 41 patients. In 34 of the 41 patients whose BAL cultures were positive, the same microorganism with the same antibiotic resistance pattern had been found earlier in the preceding routine ETA sampling. The overall primary antimicrobial treatment was adequate in $95 \%$ of the VAP cases. If the 1996 recommendations of the American Thoracic Society ${ }^{[4]}$ had been applied, only $68 \%$ ( $P=0.005)$ of the patients would have received the appropriate therapy.

Hayon et $a l,{ }^{[5]}$ in contrast, found a substantially low concordance of routine microbiological sampling. They examined 125 consecutive episodes of VAP on a medical ICU. Prior respiratory secretion cultures were available for 102 of those. The organism ultimately responsible for VAP had been recovered in routine specimens in only $36(35 \%)$ of the patients.

Similar findings are reported by Bouza et al. ${ }^{[6]}$ They obtained 1,626 respiratory surveillance samples from 356 patients on a heart surgery ICU over twelve months. Twenty-eight episodes of VAP (34.5 per 1,000 ventilation days) and 29 episodes of purulent tracheobronchitis (31.1 per 1,000 ventilation days) occurred. However, only a single episode of VAP and one tracheobronchitis were effectively predicted by surveillance cultures.

In this issue, Nair et al. ${ }^{[7]}$ present another study which shows only limited use of surveillance ETA, too. They report suspected VAP in 27 of 177 surgical ICU patients who were ventilated for at least 48 hours. But on comparing surveillance ETA cultures to clinically indicated BAL cultures during infection, only six of eleven isolates $(55 \%)$ showed an identical antimicrobial resistance pattern.

The latest infection control guidelines of the Healthcare Infection Control Practices Advisory Committee (HICPAC) of the Centers for Disease Control and Prevention (CDC) conclude that the current level of evidence on this topic does not allow recommendin routine ETA culturing for all mechanically ventilated patients. ${ }^{\left[{ }^{[8]}\right.}$ So there is a definite need for additional well designed randomized controlled trials that deal with the question of routine ETA sampling. These studies should also take the incidence of multi drug resistan bacteria into account, as these pathogens are most likely not to be covered by an empiric antimicrobial therapy approach. Besides standardized criteria and thresholds for th definition of nosocomial pneumonia should be used in studies for a better comparison of results. Further, more detailed data on the potential cost-effectiveness of ETA should also be assessed.

\section{REFERENCES}

1. Dupont $H$, Mentec $H$, Sollet JP, Bleichner G Impact of appropriateness of initial antibiotic therapy on the outcome of ventilator-associated pneumonia. Intensive Care Med 2001;27: 355-62.

2. Delclaux C, Roupie E, Blot F, Brochard L, Lemaire F, Brun-Buisson C. Lower respiratory tract colonization and infection during severe acute respiratory distress syndrome: Incidence and diagnosis. Am J Respir Crit Care Med 1997;156:1092-8.

3. Michel F, Franceschini B, Berger P, Arnal JM Gainnier M, Sainty JM, et al. Early antibiotic treatment for BAL-confirmed ventilator-associated pneumonia: A role for routine endotrachea aspirate cultures. Chest 2005;127:589-97.

4. American Thoracic Society. Hospital-acquired pneumonia in adults: Diagnosis, assessment of severity, initial antimicrobial therapy, and preventive strategies: A consensus statement, American Thoracic Society, November 1995. Am 
J Respir Crit Care Med 1996;153:1711-25.

5. Hayon J, Figliolini C, Combes A, Trouillet JL, Kassis N, Dombret MC, et al. Role of serial routine microbiologic culture results in the initial management of ventilator-associated pneumonia. Am J Respir Crit Care Med 2002;165:41-6.

6. Bouza E, Perez A, Munoz P, Jesus PM, Rincon $C$, Sanchez C, et al. Ventilator-associated pneumonia after heart surgery: A prospective analysis and the value of surveillance. Crit Care Med 2003;31:1964-70.

7. Nair S, Sen N, Peter JV, Prakash Raj J, Brahmadathan $\mathrm{KN}$. A pilot study on the role of quantitative endotracheal aspirate and cultures as a surveillance and diagnostic tool for ventilator associated pneumonia. Indian J Med Sci 2008;62:304-313.

8. Tablan OC, Anderson LJ, Besser R, Bridges C, Hajjeh R. Guidelines for preventing health-care-associated pneumonia, 2003: Recommendations of CDC and the Healthcare Infection Control Practices Advisory Committee. MMWR Recomm Rep 2004;53:1-36.

RALF-PETER VONBERG Institute for Medical Microbiology and Hospital Epidemiology, Medical School Hannover, Carl-Neuberg-Str. 1, D-30625 Hannover. E-mail: vonberg.ralf@mh-hannover.de 